\title{
Generation of Non-diffraction Vortex Beam and Its Application in Digital Communication
}

\author{
Han Huang ${ }^{1} \mathbb{D}$, Yanzhong $\mathrm{Yu}^{2}$ \\ ${ }^{1}$ Key Lab of Information Functional Materials for Fujian Higher Education, Fujian 362000, China, \\ hh4561@126.com \\ ${ }^{2}$ College of Physics \& Information Engineering, Quanzhou Normal University, Fujian 362000, China, \\ yuyanzhong059368@163.com
}

\begin{abstract}
An axicon-frustum basin-type Bessel resonator is designed for generating non-diffraction vortex beams with opposite propagation directions under different conditions. Based on the theory of geometric optics, the principle of creating vortex beams is analyzed in this axicon-frustum basin-shaped resonant cavity. It is shown that a non-diffraction vortex beam can be produced in the cavity under high-order beam stimulation. In the terahertz wavebands, the dyadic Green's function algorithm is used to numerically evaluate the electromagnetic characteristics in the cavity, and the numerical results demonstrate that the conclusion of the principle analysis is valid. Finally, the application of nondiffraction vortex beams in digital communication is explored.
\end{abstract}

Index Terms-Non-diffraction vortex beam; Resonator; Dyadic Green's function; Phase-shift keying (PSK)

\section{INTRODUCTION}

After the natural phenomenon of the vortex was found by Airy experimentally [1], its novel characteristics of singularity structure and conical surface wave have attracted much attention of a large number of researchers. In particular, the find of non-diffraction vortex beams [2],[3] has led to a series of achievements in the theory, generation, detection, transmission, and applications of vortex beams (including Bessel vortex beams). For example, it has been proven that the higher-order BesselGaussian beam [4] is a solution to the Helmholtz equation in the paraxial approximation in optics, and that it is a hollow beam with all of the properties of a vortex beam. Researchers also used the axicon to obtain the Bessel-Gaussian beam [5],[6] and explored its applications. With the continuous and indepth study of the Bessel vortex beam, its characteristics and related applications would be more fruitful. In 1995, the phenomenon that the orbital angular momentum of a photon passed to the particles was observed through experiment by $\mathrm{He}$ et al. [7]. In 1996, Simpson [8] achieved the induced rotation of tiny particles via optical vortex orbit angular momentum. This optical rotation is also named as an "optical spanner". Gahagan [9] conducted three-dimensional optical particle-bound manipulation on the particles with a vortex beam to move the particles along the focal length, creating the so-called "optical trapping technique". In addition, the vortex beam has also been widely used in 
the fields of optical computing [10], atomic cooling [11], biomedicine [12], quantum communication [13], and biological science. However, the study of vortex beam in microwave and millimeter wave bands lags far behind that of optical vortices [14],[15]. Until 2007, no one other than Thidé [16] had employed the vortex beam characteristics of orbital angular momentum (OAM) to improve the capacity of communication systems. After that, people began to get involved in this field. The vortex beam in microwave and millimeter wave bands is mainly generated by antenna arrays [17],[18],[19], helical surfaces [20],[21], and transmission grating structures [22]. This electromagnetic vortex wave with OAM provides a new degree of freedom that can be used for multiplexing technology. The value of its topological charge is unlimited in theory and its OAM is orthogonal each other, which can be widely used in wireless communication [23],[24] to expand the communication capacity. Therefore, in the present paper we reconstruct an axicon-frustum basin-shaped resonant cavity based on the original resonator. Numerical results demonstrate that the designed cavity can produce a nondiffraction vortex beam at $\mathrm{THz}$ band under high-order beam stimulation.

\section{DESIGN OF RESONATOR}

Three types of Bessel resonators [25] have been built and another type of resonator has been rebuilt in our previous work [26]. Now, an axicon-frustum basin mirror (face mirror) is added to the axiconfrustum resonator [26] to form an axicon-frustum basin-shaped resonator. As shown in Fig. 1, the cavity length of the built-in Bessel resonator satisfies the following condition:

$$
L \approx \frac{A_{1}}{2(\eta-1) \alpha}
$$

where $A_{1}$ is the aperture radius of the axicon, $\eta$ denotes the refractive index of the axicon, and $\alpha$ represents the vertex angle. The resonant cavity can oscillate to form electromagnetic modes under the condition of

$$
L=q \frac{\lambda_{q}}{2}
$$

where $L$ is the cavity length, $q$ is a positive integer, and $\lambda_{q}$ is the resonant wavelength. Fig. 1 shows an axicon-frustum basin-type Bessel resonator constructed by mirror M1 and mirror M2, where $\mathrm{M} 1$ is an axicon-frustum basin-shaped mirror and $\mathrm{M}_{2}$ is a face mirror with radius $A_{2}$. Fig. 1(a) depicts the axicon-frustum basin-shaped resonator with radius $A_{3}>A_{1} / 2$. The non-diffraction beams form the area where the two conical beams overlap each other in front of mirror $\mathrm{M}_{2}$ [26]. Fig. 1(b) shows another cavity with radius $A_{3}<A_{1} / 2$. From image theory, the beams B1 and B2 can be considered to be emitted from mirror $\mathrm{M}_{3}$ and the two beams $\mathrm{B} 1$ ' and $\mathrm{B} 2$ ' intersect in front of the mirror $\mathrm{M} 1$. $\mathrm{A}$ Bessel beam is also formed, but in the opposite direction of Fig. 1(a). If (1) and (2) are simultaneously satisfied, the cavity will oscillate to forming standing waves. If antinodes are selected on the mirror surface, a Bessel beam will appear. When a partial reflection film is plated on one mirror and a total 
reflection film is plated on another mirror, a coupled output beam can be obtained. Fig. 1(c) shows the simulation model of the axicon-frustum basin-shaped resonator.

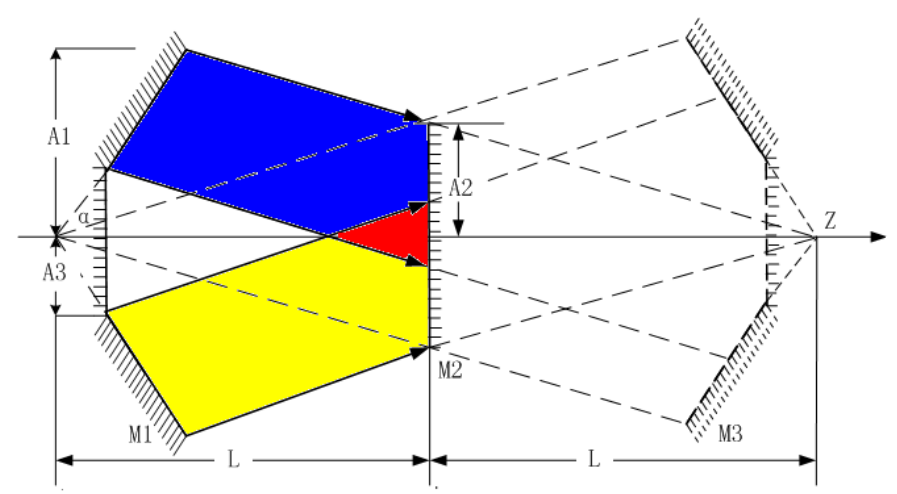

(a)

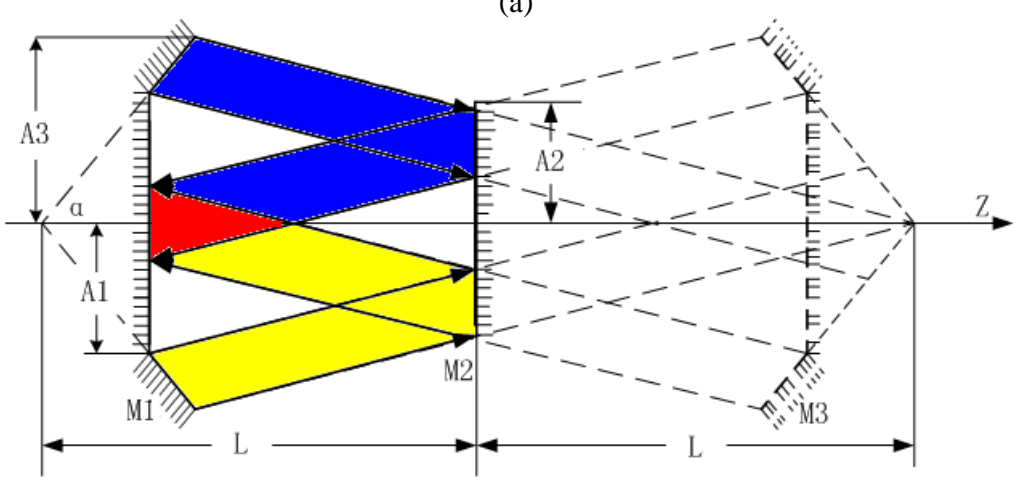

(b)

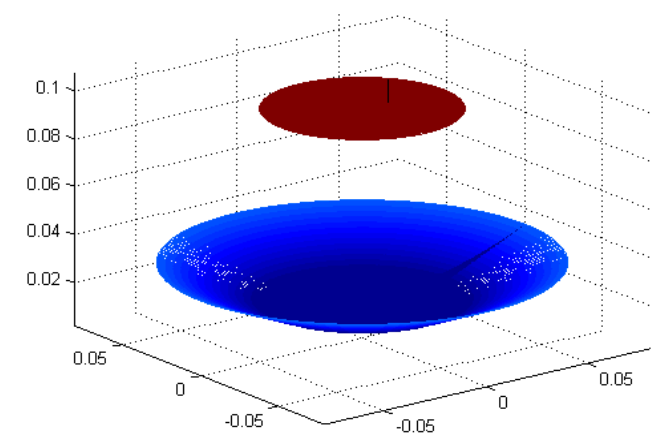

(c)

Fig.1. Axicon-frustum basin resonator with different sizes. (a) axicon-frustum basin bottom radius $A_{3}>A_{1} / 2$, (b) axiconfrustum basin bottom radius $A_{3}<A_{1} / 2$, (c) simulation model.

\section{THEORY ANALYSIS}

Suppose there is a vortex wave beam propagating along the z-axis through the spiral phase plate, satisfying:

$$
E(\rho, \varphi)=E_{0}(\rho) \exp (i 1 \varphi)
$$

where $\rho$ is the distance from the observation point to the center axis of the beam, $\varphi$ is the azimuth angle, 1 is the number of topological charge which means the mode number of the vortex beam, 
$E_{0}(\rho)$ denotes the radial amplitude distribution of incident beam. The waveform generated by the axicon is shown in Fig. 2.

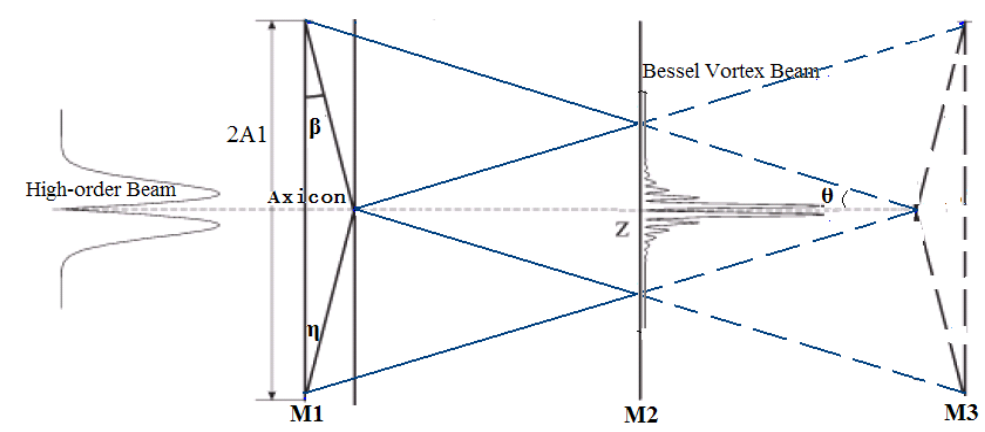

Fig. 2. Schematic diagram of the high-order Bessel vortex beam generated by the vortex beam through the axicon.

Using the Fresnel-Kirchhoff diffraction integral formula under cylindrical coordinates, the field distribution $E(\rho, z)$ after the axicon is [27],[28]:

$$
E(\rho, z)=\frac{-i k}{z} \exp \left[i k\left(z+\frac{\rho^{2}}{2 z}\right)\right] \int_{0}^{R} E\left(\rho^{\prime}, \varphi\right) t\left(\rho^{\prime}\right) J_{n}\left(\frac{k \rho \rho^{\prime}}{z}\right) \exp \left(\frac{i k \rho^{\prime 2}}{2 z}\right) \rho^{\prime} d \rho^{\prime}
$$

where $k=2 \pi / \lambda$ is the wave number in free space, $\rho$ is the radial coordinate, $R$ is the aperture radius of the axicon, $J_{n}$ is the Bessel function of the $n t h$ order, and its order is generally selected the same as the topological charge number, namely $n=1$. The penetrating rate $t(\rho)$ is written as

$$
t(\rho)=\left\{\begin{array}{c}
\exp [-i k(\eta-1) \beta \rho], \rho \leq R \\
0, \rho>R
\end{array}\right.
$$

where $\beta$ represents refraction vertex angle (see Fig. 2), and $\alpha=\arcsin (\eta \sin \beta)-\beta$. Substituting (3) and (5) into (4) yields:

$$
E(\rho, z)=\frac{-i k}{z} \exp (i k z) \int_{0}^{R} E_{0}\left(\rho^{\prime}\right) \exp (i 1 \varphi) \exp \left\{i k\left[\frac{\rho^{2}+\rho^{\prime 2}}{2 z}-(\eta-1) \beta \rho^{\prime}\right]\right\} J_{1}\left(\frac{k \rho \rho^{\prime}}{z}\right) \rho^{\prime} d \rho^{\prime}
$$

Setting $f\left(\rho^{\prime}\right)=\exp \left\{i k\left[\frac{\rho^{\prime 2}}{2 z}-(\eta-1) \beta \rho^{\prime}\right]\right\}$ yields:

$$
E(\rho, z)=\frac{-i k}{z} \exp \left[i k\left(z+\frac{\rho^{2}}{2 z}\right)\right] \int_{0}^{R} E_{0}\left(\rho^{\prime}\right) \exp (i 1 \varphi) \times J_{1}\left(\frac{k \rho \rho^{\prime}}{z}\right) \times f\left(\rho^{\prime}\right) \rho^{\prime} d \rho^{\prime}
$$

The vortex beam is confined in the non-diffractive region, as defined by the collimation $z_{\max }$ :

$$
z_{\max }=\frac{R}{(\eta-1) \theta}
$$

If the length of the conical cavity resonator satisfies $L \leq z_{\max }$, a stable non-diffracted resonated mode can be achieved in the cavity after transmitting back and forth several times. By employing the element boundary method, the field intensity of resonated mode in the cavity can be calculated by

$$
E(\rho, z)=\frac{-i k}{z} \exp \left[i k\left(z+\frac{\rho^{2}}{2 z}\right)\right] \sum_{x=\mathrm{R}}^{\mathrm{A}_{1}} E_{0}(x) \exp (i 1 \varphi) \times x J_{1}\left(\frac{k \rho x}{z}\right) \times f(x)
$$




\section{IDGF ALGORITHM AND NUMERICAL SIMULATION}

In order to accurately calculate resonant mode in the cavity, we employ the three-dimensional IDGF algorithm [25] that is the dyadic Green's function formula combined with the element boundary method:

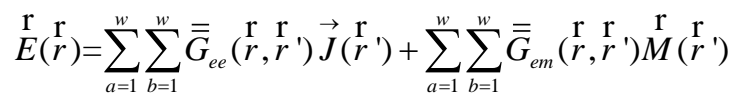

$$
\begin{aligned}
& \stackrel{\mathrm{r}}{H}(\stackrel{\mathrm{r}}{r})=\sum_{a=1}^{w} \sum_{b=1}^{w} \overline{\bar{G}}_{m e}\left(\stackrel{\mathrm{r}}{r}, \stackrel{r}{r}^{\prime}\right) \vec{J}\left(\stackrel{r}{r}^{\prime}\right)+\sum_{a=1}^{w} \sum_{b=1}^{w} \overline{\bar{G}}_{m m}(\stackrel{r}{r}, \underset{r}{r}) \stackrel{r}{M}\left(\stackrel{r}{r}^{\prime}\right)
\end{aligned}
$$

where $\stackrel{\bar{G}}{e e}_{e}\left(\stackrel{\mathrm{r}}{r}, \stackrel{\mathrm{r}}{r}^{\prime}\right), \overline{\bar{G}}_{m m}\left(\stackrel{\mathrm{r}}{r}, \stackrel{\mathrm{r}}{r}^{\prime}\right)$ denote the dyadic Green's functions of electric and magnetic types, respectively; $\bar{G}_{e m}\left(\underset{r}{r} r^{r}\right), \bar{G}_{m e}\left(\underset{r}{r}, r^{r}\right)$ denote the dyadic Green's function of electric-magnetic and magnetic-electric types, respectively; $\stackrel{1}{r}(x, y, z)$ and ${ }_{r}^{1}\left(x^{\prime}, y^{\prime} z^{\prime}\right)$ represent any observation point and source point, respectively; and $\stackrel{1}{J}(\stackrel{\mathrm{r}}{r}), \stackrel{1}{M}(\stackrel{\mathrm{r}}{r})$ represent the surface current density and surface magnetic flux density, respectively. The core steps are as follows:

Step 1: assuming that there exist an initial vortex excitation on mirror M1;

Step 2: when this excitation transits forth from mirror M1 to mirror M2, the intensity distribution on the mirror M2 can be evaluated by using Eqs. (10) and (11);

Step 3: then the beam on the M2 transits back from M2 to M1, we can also obtain the intensity distribution on the M1 in the same way;

Step 4: repeat steps 2 and 3, until the electromagnetic field in the resonator reaches to the steady state.

Step 5: The stable fields on the mirror are normalized and depicted.

As example, we present the simulation example at terahertz frequency band. The initial excitation is a high-order beam. The relevant parameters used in this example are as follows: vacuum permeability $\mu_{0}=4.0 \times \pi \times 10^{-7}$, vacuum dielectric constant $\varepsilon_{0}=8.854 \times 10^{-12}$, working wavelength $\lambda=0.32 \mathrm{~mm}, A_{1}=120 \mathrm{~mm}, A_{2}=A_{1} / 2=60 \mathrm{~mm}$, and $a=20.3845^{\circ}$. When the axicon-frustum basin radius $A_{3}=0.35 \times A_{1}$ or $A_{3}=0.75 \times A_{1}$, the topological charge $1= \pm 1$, and the distribution of the field strength at the mirror M2(M1) is shown in Fig. 3. When the radius of axicon-frustum basin $R=0.38 \times A_{1}$ or $R=0.55 \times A_{1}$, the topological charge $1= \pm 2$, and the distribution of the field strength at the mirror M2(M1) is illustrated in Fig. 4. 


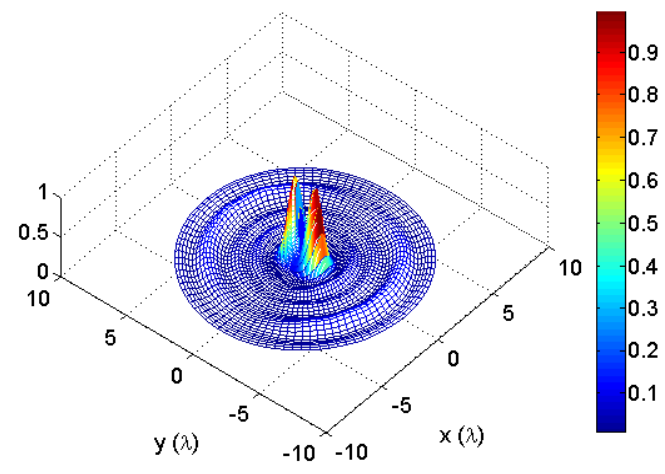

(a) $A_{3}=0.35 \times A_{1}, 1=+1$

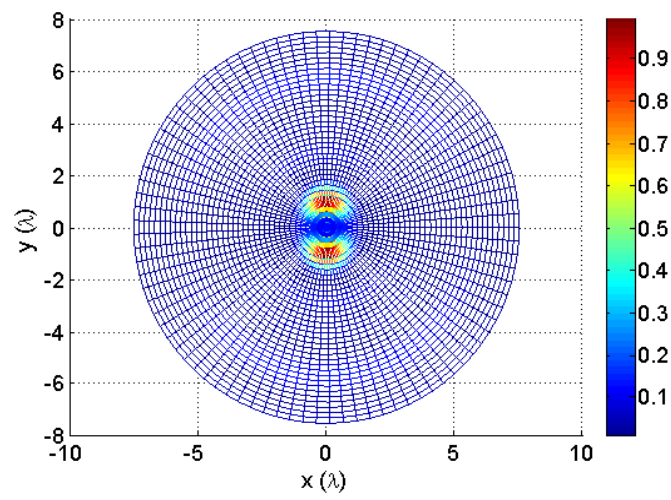

(c) $A_{3}=0.35 \times A_{1}, 1=+1$

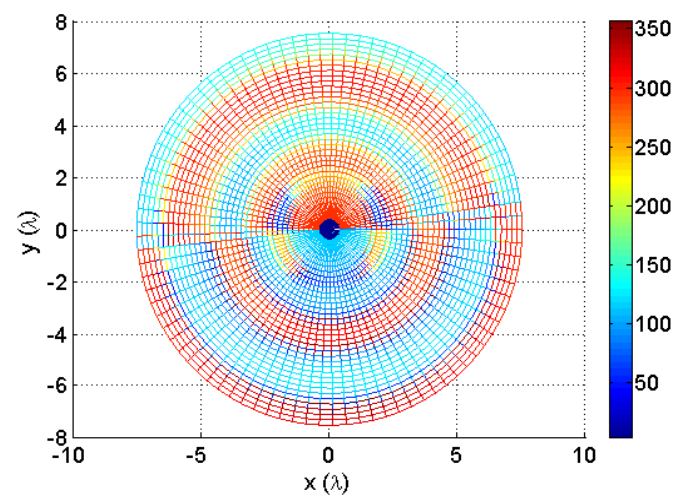

(e) $A_{3}=0.35 \times A_{1}, 1=+1$

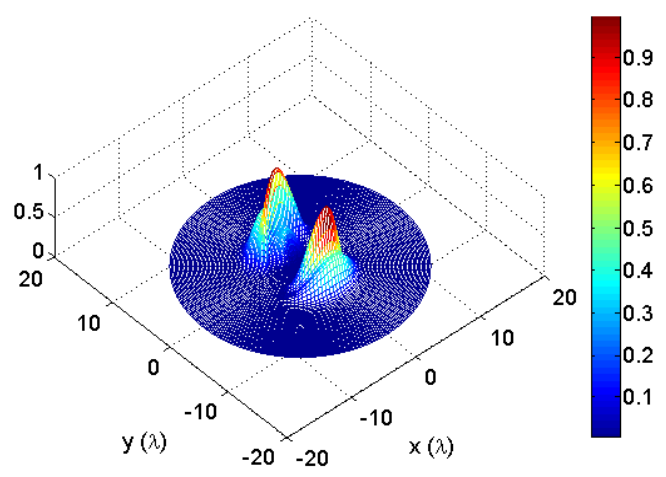

(b) $A_{3}=0.75 \times A_{1}, 1=-1$

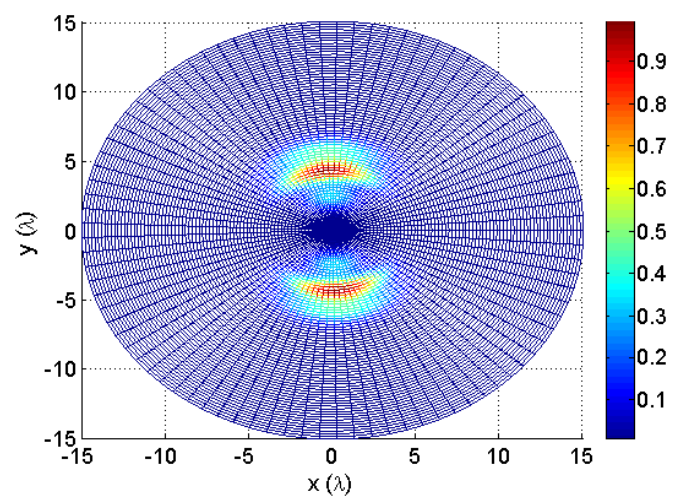

(d) $A_{3}=0.75 \times A_{1}, 1=-1$

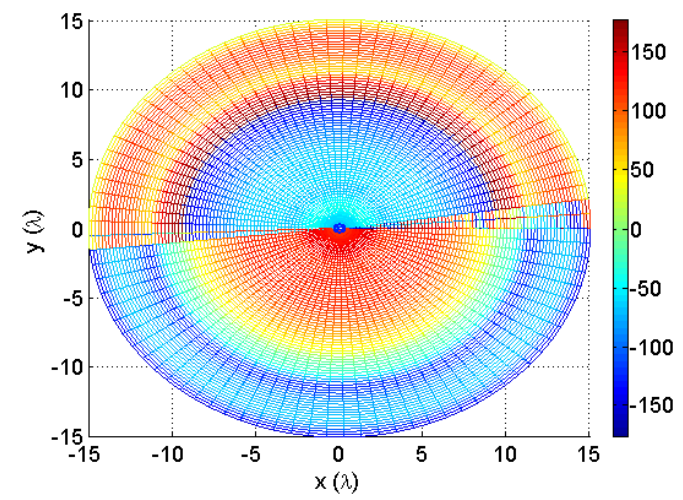

(f) $A_{3}=0.75 \times A_{1}, 1=-1$

Fig. 3. Topological charge number $1= \pm 1$, intensity and phase distributions on mirror M2 (a, c, e) and M1 (b, d, f). (a) and (b) three-dimensional (3D) normalized intensity distributions; (c) and (d) 2D normalized intensity distributions (top view); (e) and (f) 2D phase distributions. 


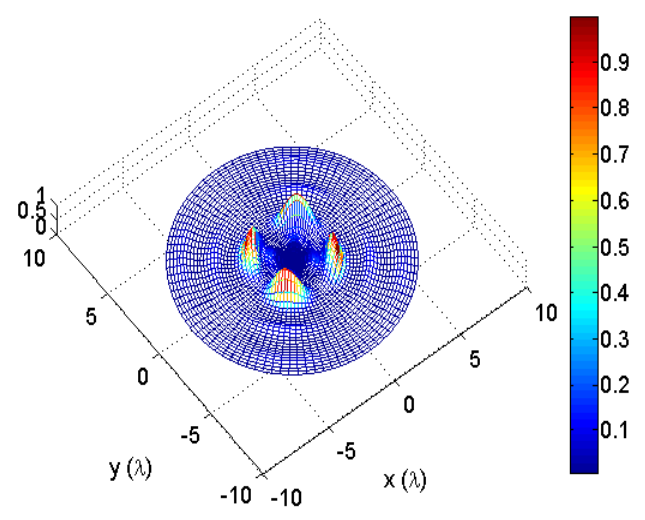

(a) $A_{3}=0.38 \times A_{1}, 1=+2$

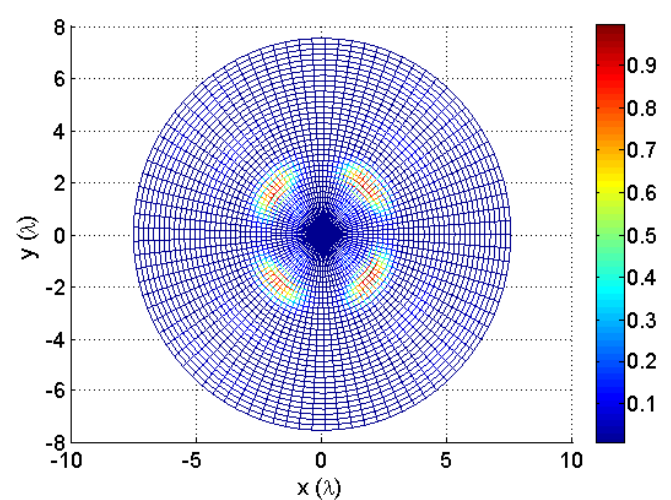

(c) $A_{3}=0.38 \times A_{1}, 1=+2$

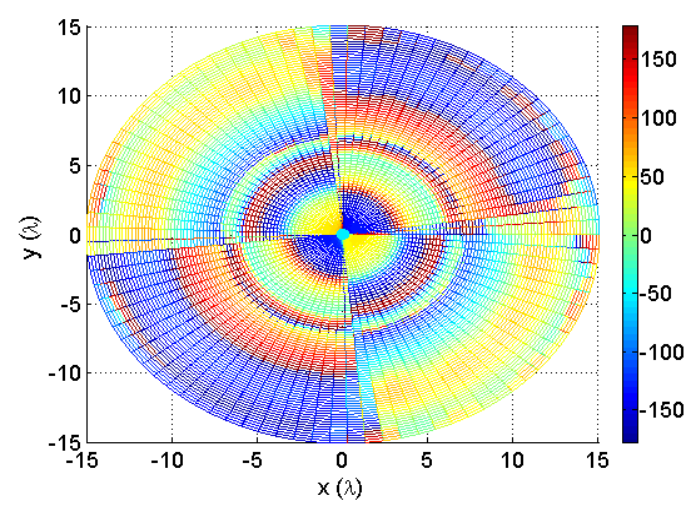

(e) $A_{3}=0.38 \times A_{1}, 1=+2$

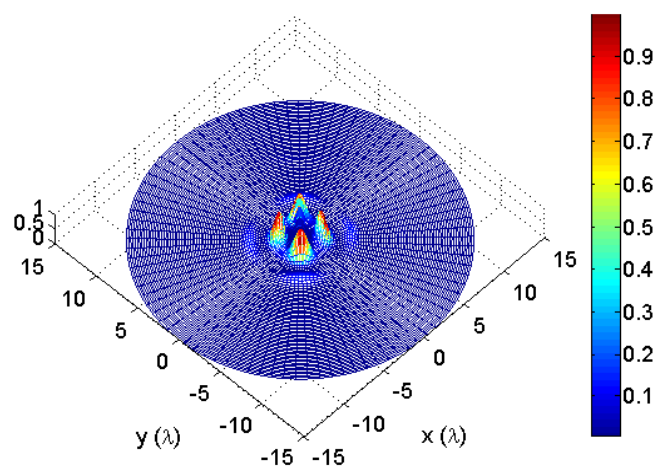

(b) $A_{3}=0.55 \times A_{1}, 1=-2$

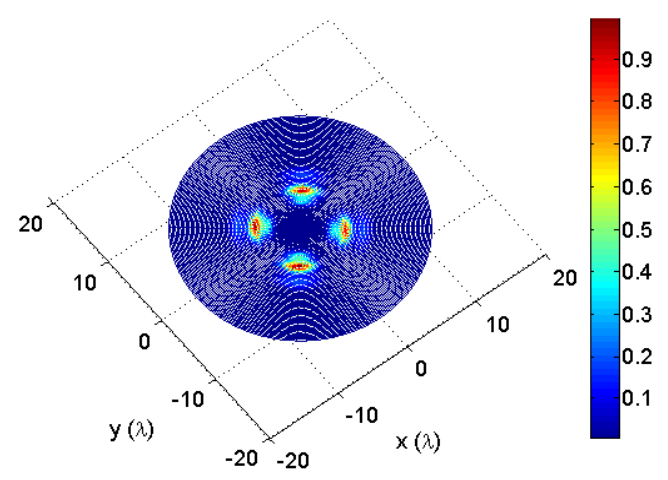

(d) $A_{3}=0.55 \times A_{1}, 1=-2$

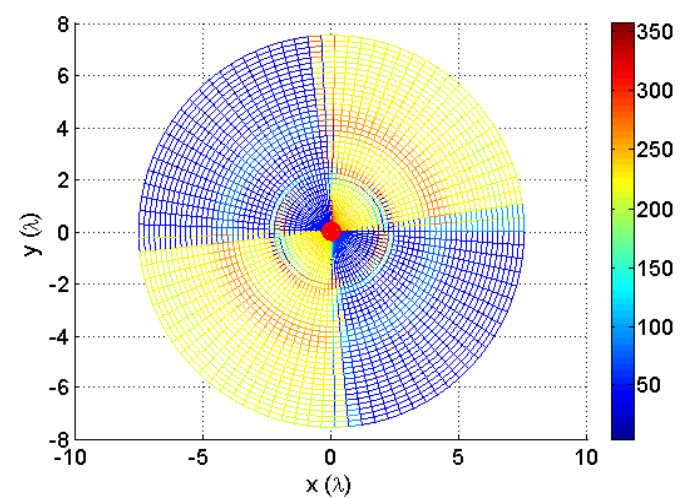

(f) $A_{3}=0.55 \times A_{1}, 1=-2$

Fig. 4. Topological charge number $1= \pm 2$, intensity and phase distributions on mirror M2 (a, c, e) and M1 (b, d, f). (a) and (b) 3D normalized intensity distributions; (c) and (d) 2D normalized intensity distributions (top view); (e) and (f) 2D phase distributions.

From this example, we can draw the following conclusions: Firstly, the oscillation can be excited to generate Bessel vortex beam in the axicon-frustum basin-type resonator at terahertz waveband. Second, the vertex angle $\alpha$ of an axicon is in proportion to the number of topological charge 1 , that is, the larger 1 generally leads to the larger $\alpha$. Third, two output beams on mirrors M1 and M2 are in the contrary directions due to the opposite directions of two mirrors. When the topological charge is $1= \pm 1$, two beams are generated and their phase difference is $180^{\circ}$; when the topological charge is Brazilian Microwave and Optoelectronics Society-SBMO received 27 July 2018; for review 7 Aug 2018; accepted 23 Apr 2019 Brazilian Society of Electromagnetism-SBMag 
$1= \pm 2$, four beams are obtained and the phase difference is $90^{\circ}$. The center of each beam is dark also named as a hollow beam. The generated Bessel vortex beam can be explored in digital communication, as discussed below.

\section{DIGITAL COMMUNICATION EXPLORATION}

A non-diffraction vortex beam with a phase singularity can play a huge role in digital communication. At present, the OAM of the vortex beam are successfully used to the multiplexing of wireless digital communication. OAM multiplexing can be performed [16] as long as the OAM mode is different, and the multiplexed total field distribution can be written by

$$
\begin{aligned}
E_{\text {multi }}(\rho, \varphi) & =\sum_{1=-2,1 \neq 0}^{2} E_{1}(\rho, \varphi) \\
& =\sum_{1=-2,1 \neq 0}^{2} E_{1}(\rho) \exp (i 1 \varphi) \\
& =E_{-2}(\rho) \exp (-i 2 \varphi)+E_{-1}(\rho) \exp (-i \varphi)+E_{+1}(\rho) \exp (i \varphi)+E_{+2}(\rho) \exp (i 2 \varphi)
\end{aligned}
$$

At the receiving end, non-diffraction beam will not spread, so the original phase rotation factor $\exp (i 1 \varphi)$ can be removed by using a spiral phase plate with the same size and topological charge of the transmitting end; thus, the ordinary electromagnetic wave can be obtained by

$$
E_{\text {demulti }}(\rho, \varphi)=\exp (-i 1 \varphi) E_{\text {multi }}(\rho, \varphi)=\exp (-i 1 \varphi) \sum_{1=-2,1 \neq 0}^{2} E_{1}(\rho) \times \exp (i 1 \varphi)
$$

For example, when $1=+2$, (13) can be rewritten as

$$
E(\rho, \varphi)=E_{-2}(\rho)+E_{-1}(\rho) \exp (i \varphi)+E_{+1}(\rho) \exp (i 3 \varphi)+E_{+2}(\rho) \exp (i 4 \varphi)
$$

It can be seen from (14) that the information carried by topological charge of $1=-2$ is obtained if omitting the last three items of (14).

In fact, the vortex beam has another feature of a fixed phase difference. This can be explored to apply in digital phase shift keying for digital communication. For instance, four different carrier phases are used to represent digital information in quaternary phase shift communication. Each carrier phase represents two bits information, as given in Table1.

TABLE I. RELATIONSHIP OF CARRIER PHASE AND TWO B
\begin{tabular}{cccc}
\hline Two-bit & symbol & \multicolumn{2}{c}{ Carrier phase } \\
\hline $\mathbf{a}$ & $\mathbf{b}$ & A mode & B mode \\
0 & 0 & 0 & $-3 \pi / 4$ \\
1 & 0 & $\pi / 2$ & $-\pi / 4$ \\
1 & 1 & $\pi$ & $3 \pi / 4$ \\
0 & 1 & $-\pi / 2$ & $\pi / 4$ \\
\hline
\end{tabular}

The quaternary PSK digital signal $\left(e_{4 p s k}\right)$ is:

$$
e_{4 p s k}=\sum_{n} g\left(t-q T_{s}\right) \cos \left(\omega_{c}+\varphi_{q}\right)
$$


where $t$ is time, $g(t)$ is the signal envelope waveform, $T_{s}$ is the symbol width, $\omega_{c}$ is the carrier frequency, and $\varphi_{q}$ denotes the corresponding phase of the qth symbol which has four values corresponding to the A or B modes. Two of them are positive, the other are negative, and the phase difference is $\pi / 2$. This resonant cavity with positive and negative output is very suitable for generating this beam. Literature [29] has proven that the propagation of vortex electromagnetic waves can reach a distance of $100 \mathrm{~km}$ in the terahertz band, so the cavity can be used 4PSK digital communication in the THZ Band, this is also our goal in the next step.

\section{SUMMARY}

In the terahertz band, the axicon-frustum basin-type Bessel resonator can be constructed using quasi-optical theory and technology. Using the principle of geometrical optics, the process of producing the non-diffraction beam in the axicon-frustum basin resonator was described simply. Applying the Fresnel-Kirchhoff diffraction integral formula, the principle of using high-order beam excitation to generate Bessel vortex beams was theoretically analyzed. Through the simulation example, we conclude that the phase distributions of the first-order mode $(1= \pm 1)$ and the secondorder mode $(1= \pm 2)$ of the non-diffraction vortex wave generated by the resonant cavity show the circular outlines of two sectors $(\varphi= \pm \pi)$ and four sectors $(\varphi= \pm \pi / 2)$, corresponding to two-vortex beams and four-vortex beams, respectively. The Bessel vortex beam generated in this paper may find application in the digital communication of PSK and its spatial multiplexing.

\section{ACKNOWLEDGEMENTS}

This work was supported by the National Natural Science Foundation of China (Grant No. 61571271), the Natural Science Foundation of Fujian Province of China (No. 2018J01646; No. 2016J01760), and the Key Discipline of Electronic Science and Technology.

\section{REFERENCES}

[1] M. Born, "Principles of Optics-electromagnetic Theory of Propagation, Interference and Diffraction of Light (7. ed.) ," DBLP, 1999.

[2] J. Durnin, M. J. Jr, J. H. Eberly, "Diffraction-free Beams," Physical Review Letters, vol. 58, no. 15, pp. 1499-1501, 2008.

[3] J. Durnin, "Exact Solutions for Non-diffracting Beams. I. The Scalar Theory," J. opt. soc, vol. 4, pp. 651-654, 1987.

[4] V. Bagini, F. Frezza,M. Santarsiero, G. Schettini, and G. Schirripaspagnolo, "Generalized Bessel-Gauss beams," Optica Acta International Journal of Optics, vol. 43, no. 6, pp. 12, 1996.

[5] G. M. Chen, H. C. Lin, J. X. Pu., "Generation of High-order Bessel Beams by Focusing Vortex Beams with an Axicon , Journal of Optoelectronics Laser, vol. 22, no. 6, pp. 945-950, 2011.

[6] X. Y. Liu, X. F. Dai, L. Y. Shan, N. Wang, and S. N. Xu, "Focusing Properties of Vortex Beam with an Axicon," Laser \& Optoelectronics Progress, vol. 49, no. 2, 022601, 2012.

[7] H. He, M. E. Friese, N. R. Heckenberg, H. Rubinsztein-Dunlop, "Direct Observation of Transfer of Angular Momentum to Absorptive Particles from a Laser beam with a Phase Singularity," Physical Review Letters, vol. 75, no. 5, pp. 826-829, 1995.

[8] N. B. Simpson, K. Dholakia, L. Allen, M. J. Padgett, "Mechanical Equivalence of Spin and Orbital Angular Momentum of Light: an Optical Spanner," Optics Letters, vol. 22, no.1, pp. 52-4, 1997.

[9] K. T. Gahagan, G. A. Swartzlander, "Optical Vortex Trapping of Particles," Optics Letters, vol. 21, no. 11, pp. 827-9, 1996.

[10] T. Kuga, Y. Torii, N. Shiokawa, T. Hirano, Y. Shimizu, and H. Sasada, "Novel Optical Trap of Atoms with a Doughnut Beam," Physical Review Letters, vol. 78, no. 25, pp.4713-4716, 1997.

[11] K. Lee, J. A. Kim, K. Kim, “Cold Atoms in Hollow Optical Systems,” Journal- Korean Physical Society, vol. 35, no. Brazilian Microwave and Optoelectronics Society-SBMO received 27 July 2018; for review 7 Aug 2018; accepted 23 Apr 2019 
3, pp. 115-121, 1999.

[12] R. Dasgupta, S. Ahlawat, R. S.Verma, P. K. Gupta, "Optical Orientation and Rotation of Trapped Red Blood Cells with Laguerre-Gaussian Mode,” Optics Express, vol. 19, no. 8, pp. 7680-7688, 2011.

[13] S. S. R. Oemrawsingh, J. A. De Jong, X. Ma, A. Aiello, E. R. Eliel, and G. W. Hooft, "High-dimensional Mode Analyzers for Spatial Quantum Entanglement,” Physical Review A, vol. 73, no. 3, 32339, 2006.

[14] J. Jin, W. Shao, F.Q Meng, "Application of Vortex Electromagnetic Wave in Military Wireless Communication," Communications Technology, vol. 47, no. 9, pp. 985-988, 2014.

[15] L.I. Xi, Z.I. Feng, J. Feng, Z. Qixun, "Electromagnetic Vortex and Its Application in Wireless Communication," Telecommunication Engineering, 2015.

[16] B. Thidé, H. Then, J. Sjöholm, K. Palmer, J. Bergman, and T. D. Carozzi, "Utilization of Photon Orbital Angular Momentum in the Low-frequency Radio Domain," Physical Review Letters, vol. 99, no. 8, $087701,2007$.

[17] D. Liu, L. Gui, M. R. Akram, "Generation of OAM Radio Waves Using Slot Antenna Array," Asia-Pacific Microwave Conference. IEEE, pp. 1-3, 2015.

[18] S. Zheng, X. Hui, X. J., H. Chi, and X. Zhang, "Generation of OAM millimeter waves using traveling-wave circular slot antenna based on ring resonant cavity," IEEE International Conference on Computational Electromagnetics, pp. 239-240, 2015.

[19] Y. LI, W. C MO, Z. G. Yang., J. S. LI, and K. J. Wang, "Generation of Terahertz Vortex Beams Based on Metasurface Antenna Array," Laser technology, vol. 41, no. 5, pp. 644-648, 2017.

[20] F. Tamburini, B. Thidé, V. Boaga, F. Carraro, M. Del Pup and A. Bianchini, "Experimental Demonstration of Freespace Information Transfer Using Phase Modulated Orbital Angular Momentum Radio," Physics, arXiv:1302.2990v2, 2013.

[21] A. Bennis, R. Niemiec, C. Brousseau, K. Mahdjoubi, and O. Emile, "Flat Plate for OAM Generation in the Millimeter Band," $7^{\text {th }}$ IEEE European Conference on Antennas and Propagat., pp. 3203-3207, 2013.

[22] F. E. Mahmouli, S. D, "Walker 4-Gbps Uncompressed Video Transmission over a $60 \mathrm{GHz}$ Orbital Angular Momentum Wireless Channel," IEEE Wireless Communications Letters, vol. 2, no. 2, pp. 223-226, 2013.

[23] F. Tamburini, E. Mari, A. Sponselli, F. Romanato, B. Thidé, and A. Bianchini, "Encoding Many Channels in the Same Frequency through Radio Vorticity: First Experimental Test," New Journal of Physics, vol. 14, no. 3, pp. 811-815, 2012.

[24] J. Kupferman, S. Arnon, "Receiver Design for OWC Orbital Angular Momentum Communication in Data Center Applications," IEEE International Symposium on Communication Systems, Networks and Digital Signal Processin., pp. 1-6, 2016.

[25] H. Huang, Y. Z. Yu. "Design and Analysis of Bessel Resonator at Terahertz Frequencies," Laser \& Optoelectronics Progress, vol. 54, no. 1, 012601, 2017.

[26] H. Huang, Y. Z. Yu. “A New Frustum Bessel Resonator,” Journal of Microwaves, vol. 33, no. 6, pp. 52-56,84, 2017.

[27] V. Jarutis, R. Paškauskas, A. Stabinis. "Focusing of Laguerre-Gaussian Beams by Axicon," Optics Communications, vol. 184, no. 1-4, pp.105-112, 2000.

[28] G. M. Chen, H. C. Lin, J. X. Pu, "Generation of High-order Bessel Beams by Focusing Vortex Beams with an Axicon," Journal of Optoelectronics Laser, vol. 22, no. 6, pp. 945-950, 2011.

[29] Z. F. Lv, L. K. Lin, C. S. Lu, "Research on the Characteristics of Vortex Electromagnetic Wave Transmission in Terahertz Band," National Conference on Microwave Millimeter Wave (ZhongCe). China, 2017. 